\title{
Diferenças nos Padrões de Tratamento e nas Características Epidemiológicas entre Pacientes Idosas e Adultas Portadores de Câncer do Colo do Útero
}

Differences in Treatment Patterns and Epidemiological Characteristics between Elderly and Adult Patients with Cervical Cancer

Las Diferencias en los Patrones de Tratamiento y en las Características Epidemiológicas entre las Pacientes Mayores y Adultas con Cáncer del Cuello del Útero

\author{
Mariana do Nascimento Vilaça ${ }^{1}$; Márcio Luiz Martins Junior ${ }^{2}$ Luciano Rios Scherrer ${ }^{3}$; Angélica Nogueira-Rodrigues ${ }^{4}$
}

\begin{abstract}
Resumo
Introduçáo: $\mathrm{O}$ avanço da idade associa-se a menores taxas de sobrevida e é importante fator para seleção do tratamento do câncer. Objetivos: Avaliar as características epidemiológicas e diferenças existentes entre os tratamentos realizados em mulheres idosas (idade igual ou maior que 60 anos) com câncer do colo do útero, comparados àqueles referentes a pacientes adultas (idade menor que 60 anos). Método: Estudo retrospectivo e descritivo no qual foram analisados os prontuários das pacientes diagnosticadas com carcinoma do colo do útero entre 1999 e 2010. Os prontuários foram separados em dois grupos: idosas, com 60 anos ou mais, e adultas, com menos de 60 anos. Os dados coletados foram salvos em planilhas do programa Microsoft Office Excel 2003. As características clínicas e epidemiológicas coletadas foram comparadas utilizando o teste qui-quadradro. $\mathrm{O}$ valor de $\mathrm{p}$ considerado significante foi menor que 0,05. Resultados: Entre 67 pacientes idosas (40,12\%) e 100 adultas (59,88\%), observou-se uma maior incidência de tumores avançados IIB e III nas idosas, enquanto estágios iniciais in situ, IB e IIA incidiram mais entre as adultas $(\mathrm{p}<0,05)$. As adultas estadiadas em IB, IIA e III foram mais submetidas a procedimentos cirúrgicos, radioterapia e quimioterapia, enquanto nas idosas o tratamento mais comum foi a radioterapia isolada $(p<0,05)$. Conclusáo: Essa análise mostra a relevância do câncer de colo do útero em idosas, no que concerne à alta frequência, estágios mais avançados ao diagnóstico e estratégias menos agressivas de tratamento. Há necessidade de programas de rastreamento e estudos clínicos direcionados a este grupo populacional.
\end{abstract}

Palavras-chave: Neoplasias do Colo do Útero/terapia; Perfil de Saúde; Idoso; Estudos Retrospectivos; Epidemiologia Descritiva

\footnotetext{
${ }^{1}$ Graduanda de Medicina. Faculdade de Medicina da Universidade de Itaúna. Itaúna (MG), Brasil. http://www.uit.edu.br. Hospital São João de Deus, Centro de Assistência de Alta Complexidade em Oncologia (CACON) do Centro-Oeste de Minas Gerais. Divinópolis (MG), Brasil. http://www.hsjd.com.br. E-mail: marianavilaca@yahoo.com.br.

${ }^{2}$ Graduando de Medicina. Faculdade de Medicina da Universidade de Itaúna. Itaúna (MG), Brasil. Hospital São João de Deus, CACON do Centro-Oeste de Minas Gerais. Divinópolis (MG), Brasil. http://www.hsjd.com.br. E-mail: marcioluizmartins@hotmail.com.

${ }^{3}$ Estatístico da Sociedade Brasileira de Oncologia Clínica. Mestre em Estatística pela Universidade Federal de Minas Gerais (UFMG). Belo Horizonte (MG), Brasil. http://bioestatístico@sboc.org.br.

${ }^{4}$ Médica Oncologista. Mestre em Saúde da Mulher pela UFMG. Doutoranda em Oncologia Clínica pelo INCA. Oncologista da Pesquisa Clínica do INCA e CACON Centro-Oeste de Minas Gerais. Divinópolis (MG), Brasil. http://www.hsjd.com.br.

Endereço para correspondência: Angélica Nogueira-Rodrigues. Rua 23 de novembro, 570, Niteroi. Divinópolis (MG), Brasil. CEP: $35500-249$.

E-mail: angelica.onco@uol.com.br.
} 


\section{INTRODUÇÃO}

Apesar do crescente avanço na prevenção e tratamento do câncer do colo do útero, ainda é grande a morbimortalidade causada pela doença. Essa neoplasia segue sendo a terceira causa de câncer e a quarta causa de mortes por câncer em mulheres, correspondendo a cerca de $10 \%$ dos novos casos e $8 \%$ do total de óbitos. Especialmente nas áreas em desenvolvimento do mundo, onde estão concentrados mais de $85 \%$ dos casos, o câncer do colo do útero apresenta-se como grave problema de saúde pública ${ }^{1-2}$.

No Brasil, segundo estimativa do Instituto Nacional de Câncer José Alencar Gomes da Silva (INCA), para 2012, são esperados 17.540 novos casos de câncer do colo do útero, com risco estimado de 17 casos por $100 \mathrm{mil}$ mulheres, ou 9,3\% do total, constituindo-se o segundo mais frequente nesta população, excluindo-se tumores de pele não melanoma ${ }^{1}$.

A taxa de mortalidade por esse câncer no Brasil, no ano de 2007, foi de aproximadamente 5,2 óbitos por 100 mil mulheres $^{3}$. Nos últimos anos, observou-se uma tendência de declínio dessa taxa, tanto nas capitais como nos municípios do interior, sendo a queda maior nas primeiras. As maiores taxas se encontram nas Regiōes Norte, Nordeste e Centro-Oeste e, em alguns municípios do interior do Norte e Nordeste, houve um aumento da mortalidade. Apesar da diminuição nas taxas de mortalidade por câncer do colo do útero, estas ainda podem ser consideradas altas, quando comparadas com as de países mais desenvolvidos ${ }^{4}$.

Desde 1992, a Organização Mundial da Saúde considera que a persistência de altas cargas virais do Papilomavirus humano (HPV) representa o principal fator de risco para o desenvolvimento do câncer do colo do útero. A infecção pelo HPV é transmitida sexualmente e, segundo estudos moleculares e epidemiológicos, está fortemente associada ao desenvolvimento de neoplasias nas células cervicais. Pesquisas que utilizam métodos de hibridização têm demonstrado que mais de $99 \%$ dos casos podem ser atribuídos a algum tipo de HPV. Sabe-se que a infecção pelo HPV é essencial, mas não o suficiente para o desenvolvimento desse câncer ${ }^{5-8}$.

O pico de incidência do câncer do colo do útero situa-se entre a quinta e sexta décadas de vida, com as taxas de mortalidade registrando aumento com o avançar da idade em todo país ${ }^{1,3}$. Segundo dados do Sistema de Informaçóes sobre Mortalidade (SIM), em 2009, dos 5.063 óbitos por câncer do colo do útero, 2.124 foram em mulheres com 60 anos ou mais, correspondendo a $41,95 \%$ das mortes por esse tumor. No entanto, até o ano de 2011, o exame Papanicolaou era preconizado pelo Programa Nacional de Controle do Câncer do Colo do Útero e de Mama - Viva Mulher, apenas para mulheres até os 59 anos de idade ${ }^{3,9}$.
Segundo dados desse mesmo programa, em Minas Gerais, no ano de 2010, a razão de exames citopatológicos para rastreamento do câncer do colo do útero para a população feminina de 25 a 59 anos foi de 0,20 ; enquanto, para as pacientes com 60 anos ou mais, essa razáo foi de 0,09 . No município de Divinópolis, na Região Centro-Oeste de Minas Gerais, essa mesma taxa para as pacientes de 25 a 59 anos foi de 0,14 e para as pacientes com 60 anos ou mais foi de $0,09^{10}$.

Apesar de o câncer do colo do útero ser relativamente frequente em mulheres idosas, o impacto da idade na sobrevida da paciente ainda é incerto. $\mathrm{O}$ avanço da idade está associado a um decréscimo na sobrevida e tem-se mostrado um importante fator para seleção do tratamento contra o câncer. As pacientes idosas geralmente são submetidas a tratamentos menos agressivos, quando comparadas com pacientes mais jovens ${ }^{11}$.

O tratamento para o câncer do colo do útero apresenta variaçóes de acordo com o estadiamento da doença, tamanho do tumor, condiçôes clínicas da paciente e se esta apresenta prole definida. Os tumores em estágios iniciais, de IA a IIA, são tratados preferencialmente com procedimentos cirúrgicos. $\mathrm{Na}$ contraindicação deste, as pacientes são tratadas com radioterapia exclusiva. $\mathrm{O}$ tratamento dos tumores localmente avançados é feito através da associação de quimioterapia com cisplatina e radioterapia. Esse tipo de tratamento foi proposto a partir de 1999, com a comprovação de aumento na sobrevida com a incorporação de quimioterapia com cisplatina ao tratamento radioterápico. Atualmente, essa associação é indicada também para o tratamento de tumores estadiados em IB e IIA com comportamento mais agressivo. As cirurgias em tumores localmente avançados são paliativas, utilizadas principalmente no estágio IVA, indicadas de acordo com as condiçóes e necessidades individuais da paciente. Os tumores estadiados em IVB são considerados incuráveis, sendo o tipo de tratamento paliativo indicado de acordo com as necessidades de cada paciente, com intuito de melhorar sua qualidade de vida ${ }^{12-14}$.

Baseado nesse panorama, este estudo tem como objetivo avaliar as características epidemiológicas e diferenças existentes entre os tratamentos realizados em mulheres idosas (idade igual ou maior que 60 anos) com câncer do colo do útero, comparadas àquelas referentes a pacientes adultas (idade menor que 60 anos), tratadas no Centro de Assistência de Alta Complexidade em Oncologia (CACON) do Centro-Oeste de Minas Gerais, na cidade de Divinópolis.

\section{MÉTODO}

A partir da aprovação do estudo pelo Comitê de Ética em Pesquisa (CEP), com número de registro do protocolo de 222, realizou-se um estudo retrospectivo e 
descritivo através da análise de prontuários de pacientes diagnosticadas com câncer do colo do útero e tratadas no CACON do Centro-Oeste de Minas Gerais.

Separou-se esses prontuários em dois grupos, o primeiro com pacientes idosas, consideradas aquelas com idade igual ou maior que 60 anos e o segundo com pacientes adultas, com idade menor que 60 anos, de acordo com a idade apresentada no diagnóstico anatomopatológico constante no prontuário. A idade de 60 anos foi escolhida devido ao fato de, até o ano de 2011, o programa Viva Mulher preconizar o rastreamento para câncer do colo do útero apenas para mulheres até 59 anos.

Em seguida, realizou-se a coleta dos seguintes dados: idade da paciente na data do diagnóstico anatomopatológico, data do diagnóstico anatomopatológico, histologia do tumor, grau de diferenciação histopatológico, estadiamento do tumor de acordo com a Federaçáo Internacional de Ginecologia e Obstetrícia (FIGO), tratamentos para os quais a paciente foi alocada, comorbidades, peso, altura, índice de massa corporal (IMC), número de gestaçôes, raça, estado civil e profissão. Os tumores foram classificados histologicamente em carcinoma de células escamosas, adenocarcinoma, carcinoma adenoescamoso, adenocarcinoma de células claras e tumores indiferenciados. $\mathrm{O}$ grau de diferenciação dos tumores invasivos foi definido como: bem diferenciado (G1), moderadamente diferenciado (G2), pouco diferenciado (G3) e indiferenciado (G4). Em relação aos tratamentos, estes foram classificados em cirurgia, radioterapia, sendo as modalidades externa e braquiterapia consideradas em conjunto na modalidade radioterapia, quimioterapia, associaçóes entre esses tratamentos ou nenhum tratamento. Na categoria cirurgia, considerou-se como procedimentos cirúrgicos as histerectomias e suas variaçôes, e traquelectomia. Aquelas pacientes que foram submetidas à laparotomia, mas a histerectomia não pôde ser realizada devido à grande extensáo do tumor ou aquelas que foram histerectomizadas por outra finalidade que não o tratamento do câncer, foram classificadas nos outros grupos de tratamentos propostos aos quais as pacientes foram alocadas. Os tratamentos considerados foram aqueles em que a paciente foi alocada primariamente, logo após o diagnóstico, sem considerar para análise, tratamentos subsequentes. O IMC foi obtido a partir dos primeiros dados de peso e altura constantes no prontuário, ao início do tratamento da paciente.

Os dados foram coletados em formulários impressos e posteriormente passados e salvos em planilhas do programa Microsoft Office Excel 2003. As características clínicas e epidemiológicas coletadas foram comparadas utilizando o teste qui-quadradro. O valor de p, considerado estatisticamente significante, foi menor do que 0,05. A análise foi feita na procura de diferenças nos tratamentos propostos e nas características das pacientes idosas com câncer do colo do útero.

\section{RESULTADOS}

Foram analisados 167 prontuários com período de diagnósticos entre 16 de outubro de 1999 a 26 de agosto de 2010. Nestes, 67 pacientes tinham 60 anos ou mais, correspondendo a $40,12 \%$, e 100 possuíam idade inferior a 60 anos ou $59,88 \%$ do total. A idade das pacientes idosas variou entre 60 a 83 anos e das pacientes adultas situou-se entre 17 e 59 anos (Tabela 1).

A análise estatística mostrou que o estado civil de casada foi mais comum entre as adultas e as viúvas foram mais frequentes no grupo idoso $(\mathrm{p}<0,05)$. Não foi observada diferença para a incidência da doença entre as raças $(p>0,05)$. Em relação à profissão, a maior parte das mulheres dos dois grupos designou-se como do lar (Tabela 1).

Em relação à histologia do tumor, o carcinoma de células escamosas foi o mais frequente em ambos os grupos, ocorrendo em 64 pacientes adultas (64\%) e 54 idosas $(80,6 \%)$. Não foi observada maior incidência de algum tipo histológico de tumor entre adultas ou idosas $(p>0,05)$, assim como não foi houve diferença estatística entre os grupos no que concerne à incidência das diferentes graduaçôes histopatológicas ( $p>0,05)$ (Tabela 2).

Tabela 1. Características sociodemográficas

\begin{tabular}{|c|c|c|c|}
\hline Variáveis & $\begin{array}{c}\text { Até } 59 \\
\text { anos } \\
n=100\end{array}$ & $\begin{array}{l}60 \text { anos } \\
\text { ou mais } \\
N=67\end{array}$ & $\begin{array}{l}\text { Valor } \\
\text { de p }\end{array}$ \\
\hline \multicolumn{4}{|l|}{ Idade } \\
\hline Média & 46,1 & 69,4 & \\
\hline Mediana & 47 & 69 & \\
\hline Desvio-padrão & 9,89 & 7,14 & \\
\hline \multicolumn{4}{|l|}{ Raça } \\
\hline Branca & $14(14 \%)$ & $6(8,96 \%)$ & \multirow{4}{*}{$p>0,05$} \\
\hline Parda & $6(6 \%)$ & $5(7,46 \%)$ & \\
\hline Negra & 7 (7\%) & $3(4,48 \%)$ & \\
\hline Sem dados & $73(73 \%)$ & $53(79,1 \%)$ & \\
\hline \multicolumn{4}{|l|}{ Estado Civil } \\
\hline Casada & $30(30 \%)$ & $8(11,9 \%)$ & \multirow{5}{*}{$p<0,05$} \\
\hline Solteira & $9(9 \%)$ & $1(1,5 \%)$ & \\
\hline Viúva & $12(12 \%)$ & $18(26,9 \%)$ & \\
\hline $\begin{array}{l}\text { Divorciada/ } \\
\text { desquitada }\end{array}$ & $2(2 \%)$ & $2(3 \%)$ & \\
\hline Sem dados & 47 (47\%) & $38(56,7 \%)$ & \\
\hline \multicolumn{4}{|l|}{ Profissão } \\
\hline Do lar & $22(22 \%)$ & $10(14,9 \%)$ & \multirow{4}{*}{$p>0,05$} \\
\hline Aposentada & $2(2 \%)$ & $3(4,5 \%)$ & \\
\hline Outros & $14(14 \%)$ & $2(3 \%)$ & \\
\hline Sem dados & $62(62 \%)$ & $52(77,6 \%)$ & \\
\hline
\end{tabular}


Vilaça MN, Martins Junior ML, Scherrer LR, Nogueira-Rodrigues A

Tabela 2. Características da doença e outros indicadores de saúde

\begin{tabular}{|c|c|c|c|}
\hline Variáveis & $\begin{array}{c}\text { Até } 59 \text { anos } \\
n=100\end{array}$ & $\begin{array}{c}60 \text { anos ou mais } \\
N=67\end{array}$ & Valor de $p$ \\
\hline \multicolumn{4}{|l|}{ Histologia do tumor } \\
\hline Tumor in situ & $16(16 \%)$ & $3(4,48 \%)$ & \multirow{6}{*}{$p>0,05$} \\
\hline Escamoso & $64(64 \%)$ & $54(80,6 \%)$ & \\
\hline Adenocarcinoma & $17(17 \%)$ & $9(13,43 \%)$ & \\
\hline Adenoescamoso & $1(1 \%)$ & 0 & \\
\hline Células claras & $2(2 \%)$ & 0 & \\
\hline Indiferenciado & 0 & $1(1,49 \%)$ & \\
\hline \multicolumn{4}{|c|}{ Grau de diferenciação (exceto tumores in situ) } \\
\hline I & $4(4 \%)$ & $4(5,97 \%)$ & \multirow{5}{*}{$p>0,05$} \\
\hline II & $23(23 \%)$ & $19(28,36 \%)$ & \\
\hline III & $15(15 \%)$ & $20(29,85 \%)$ & \\
\hline IV & 0 & $1(1,49 \%)$ & \\
\hline Não determinado & $42(42 \%)$ & $20(29,85 \%)$ & \\
\hline \multicolumn{4}{|l|}{ Estadiamento FIGO } \\
\hline 0 & $16(16 \%)$ & $3(4,5 \%)$ & \multirow{6}{*}{$p<0,05$} \\
\hline $\mathrm{IB} / \mathrm{IIA}$ & $31(31 \%)$ & $14(20,9 \%)$ & \\
\hline IIB & $21(21 \%)$ & $26(38,8 \%)$ & \\
\hline III & 17 (17\%) & $18(26,9 \%)$ & \\
\hline IV & $6(6 \%)$ & $1(1,5 \%)$ & \\
\hline Não Estadiadas & $9(9 \%)$ & $5(7,5 \%)$ & \\
\hline \multicolumn{4}{|c|}{ Presença de Comorbidades } \\
\hline Sim & 31 (31\%) & $32(47,7 \%)$ & \multirow{3}{*}{$p<0,05$} \\
\hline Não & $11(11 \%)$ & $6(9,0 \%)$ & \\
\hline Sem dados & $58(56 \%)$ & $29(43,3 \%)$ & \\
\hline \multicolumn{4}{|c|}{ Principais Comorbidades } \\
\hline HAS & 20 & 25 & \multirow{7}{*}{$p<0,05$} \\
\hline DM & 9 & 9 & \\
\hline Problemas cardíacos & 0 & 4 & \\
\hline Problemas na tireoide & 4 & 2 & \\
\hline Outras neoplasias & 0 & 3 & \\
\hline Hidronefrose & 6 & 2 & \\
\hline Outras & 5 & 5 & \\
\hline \multicolumn{4}{|c|}{ Índice de Massa Corporal (IMC) } \\
\hline$<18$ & $3(3 \%)$ & $9(13,4 \%)$ & \multirow{5}{*}{$\mathrm{p}<0,05$} \\
\hline De 18 a 24,9 & $26(26 \%)$ & $20(29,9 \%)$ & \\
\hline De 25 a 30 & $25(25 \%)$ & $6(9,0 \%)$ & \\
\hline$>30$ a 39,9 & 11 (11\%) & $8(11,9 \%)$ & \\
\hline Sem dados & 35 (35\%) & $24(35,8 \%)$ & \\
\hline \multicolumn{4}{|l|}{ Gestações } \\
\hline Média & 2,22 & 3,31 & \\
\hline Desvio-padrão & 2,82 & 4,51 & \\
\hline
\end{tabular}


A análise do estadiamento dos tumores mostrou que as pacientes adultas são diagnosticadas em estágios mais precoces da doença, considerando, tumores in situ e tumores invasivos IB e IIA, quando comparadas com as pacientes idosas $(\mathrm{p}<0,05)$. Quarenta e sete por cento das pacientes adultas foram diagnosticadas em estágios inicias do câncer do colo do útero, com tumores in situ ou estágios IB e IIA, enquanto, entre as pacientes mais velhas, essa porcentagem foi de $25,4 \%(\mathrm{p}<0,05)$ (Tabela 2$)$.

Em relação ao IMC, houve uma maior frequência de pacientes idosas com valores de IMC abaixo do considerado normal, de $18 \mathrm{Kg} / \mathrm{m}^{2}$. As pacientes adultas mais frequentemente apresentaram-se com sobrepeso, com valores de IMC de 25 a $30 \mathrm{Kg} / \mathrm{m}^{2}(\mathrm{p}<0,05)$. As comorbidades não foram mais frequentes em um dos grupos estudados $(p>0,05)$, sendo que a mais prevalente nos dois grupos foi a Hipertensão Arterial Sistêmica (HAS), atingindo 25 idosas (37,31\%) e 20 adultas (20\%) (Tabela 2).

No tratamento dos tumores in situ, ambos os grupos foram tratados em quase sua totalidade com procedimentos cirúrgicos, não se observando diferença quanto aos tratamentos propostos para idosas e adultas (p>0,05) (Tabela 3).
Nos estágios IB e IIA, as pacientes adultas foram mais comumente submetidas a procedimentos cirúrgicos em associação com radioterapia e/ou quimioterapia $(\mathrm{p}<0,05)$. Essa modalidade de tratamento foi utilizada em 25 pacientes adultas $(80,6 \%)$ estadiadas em IB ou IIA e 6 idosas (42,9\%) (Tabela 3).

No que concerne aos tratamentos realizados em pacientes diagnosticadas em estágio IIB não se observou diferença estatística $(p>0,05)$. Em ambos os grupos, o tratamento mais utilizado foi a associaçáo de radioterapia e quimioterapia, sendo este realizado em nove pacientes adultas (42,8\%) e oito pacientes idosas (30,8\%) (Tabela 3).

Em relação às pacientes com estágio III, constatou-se diferença quanto aos tratamentos utilizados para pacientes adultas quando comparadas com aquelas com 60 anos ou mais $(\mathrm{p}<0,05)$. Entre as pacientes com menos de 60 anos, o tratamento cirúrgico associado à radioterapia e/ou quimioterapia foi o mais comumente realizado, enquanto, para as pacientes idosas, o tratamento mais frequente foi a radioterapia isolada (Tabela 3 ).

Não foram observadas diferenças estatísticas entre os tratamentos realizados para os dois grupos entre as pacientes estadiadas em IV, grupo no qual o tratamento mais utilizado foi a radioterapia isolada (Tabela 3) .

Tabela 3. Tratamentos indicados por estadiamento da doença

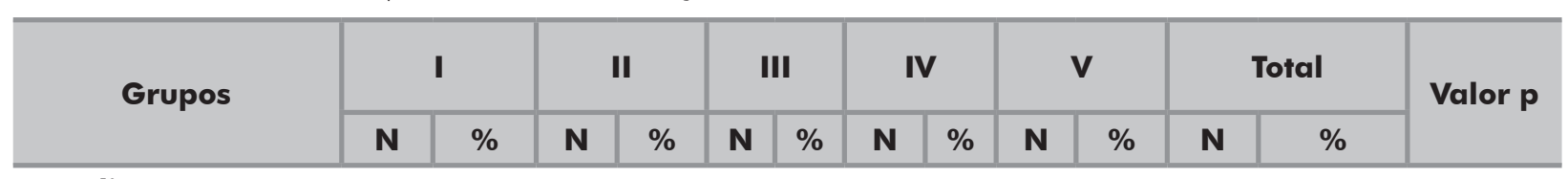

Estadiamento: Tumores in situ

\begin{tabular}{l|c|c|c|c|c|c|c|c|c|c|c|c|c|c}
\hline Jovens & - & - & 14 & 87,5 & - & - & - & - & 2 & 12,5 & 16 & 100 & 0,517 \\
\hline Idosas & - & - & 3 & 100 & - & - & - & - & 0 & 0 & 3 & 100 & \\
\hline Estadiamento: IB/IIA & 2 & 6,5 & 3 & 9,7 & 25 & 80,6 & 0 & 0 & 1 & 3,2 & 31 & 100 & \multirow{2}{*}{0,015} \\
\hline Jovens & 5 & 35,7 & 1 & 7,1 & 6 & 42,9 & 2 & 14,3 & 0 & 0 & 14 & 100 & 0,015 \\
\hline Idosas & 5
\end{tabular}

Estadiamento: IIB

\begin{tabular}{|c|c|c|c|c|c|c|c|c|c|c|c|c|c|}
\hline Jovens & 6 & 28,6 & - & - & 6 & 28,6 & 9 & 42,8 & - & - & 21 & 100 & \multirow{2}{*}{0,329} \\
\hline Idosas & 13 & 50 & - & - & 5 & 19,2 & 8 & 30,8 & - & - & 26 & 100 & \\
\hline \multicolumn{14}{|c|}{ Estadiamento: III } \\
\hline Jovens & 7 & 41,2 & - & - & 7 & 41,2 & 3 & 17,6 & - & - & 17 & 100 & \multirow{2}{*}{0,008} \\
\hline Idosas & 14 & 77,8 & - & - & 0 & 0 & 4 & 22,2 & - & - & 18 & 100 & \\
\hline \multicolumn{14}{|c|}{ Estadiamento IV } \\
\hline Jovens & 3 & 50 & 1 & 16,7 & 1 & 16,7 & 1 & 16,7 & - & - & 6 & 100 & \multirow{2}{*}{0,831} \\
\hline Idosas & 1 & 100 & 0 & 0 & 0 & 0 & 0 & 0 & - & - & 1 & 100 & \\
\hline
\end{tabular}

\section{Sem Estadiamento}

\begin{tabular}{l|c|c|c|c|c|c|c|c|c|c|c|c|c}
\hline Jovens & 2 & 22,2 & 0 & 0 & 5 & 5,56 & 1 & 11,1 & 1 & 11,1 & 9 & 100 & - \\
\hline Idosas & 1 & 20 & 2 & 40 & 2 & 40 & 0 & 0 & 0 & 0 & 5 & 100 & \\
\hline
\end{tabular}

I - Radioterapia (externa e/ou interna); II - Tratamento cirúrgico isolado; III - Procedimento cirúrgico associado a radioterapia e/ou quimioterapia; IV - Associaçăo de radioterapia e quimioterapia; $\mathrm{V}$ - Sem tratamento 


\section{DISCUSSÃO}

A partir de 1999, a evidência de aumento na sobrevida levou à mudança do tratamento do câncer do colo do útero localmente avançado, sendo adicionada quimioterapia baseada em cisplatina à radioterapia externa e braquiterapia. No entanto, as pacientes idosas são mais frequentemente submetidas à radioterapia isolada do que as pacientes adultas. Assim, há evidência de que a idade interfere na escolha terapêutica, mas isso tem impacto negativo na sobrevida de pacientes com câncer ${ }^{11,15}$.

Quando submetidas à quimioterapia associada à radioterapia e braquiterapia, as pacientes idosas apresentam taxas de sobrevida e de complicaçóes semelhantes às das pacientes mais jovens, dado que alerta que a idade em si não deve constituir em contraindicação para tratamentos mais agressivos em pacientes mais velhas ${ }^{11,14-17}$. Além disso, as pacientes idosas têm resposta à radioterapia e braquiterapia semelhantes a pacientes adultas ${ }^{18-19}$. Um estudo com coorte histórica também demonstrou que a histerectomia radical do tipo Wertheim é segura para pacientes adultas e idosas com 60 anos ou mais. Nessa coorte, não houve diferença na mortalidade e morbidade após a cirurgia entre os dois grupos ${ }^{19}$.

Os resultados do presente estudo também evidenciam que os padróes de cuidado para as pacientes idosas portadoras de câncer do colo do útero diferem de maneira importante. As pacientes adultas, nos estágios iniciais da doença entre IB e IIA e com tumores localmente avançados em estágio III, foram significativamente mais submetidas a procedimentos cirúrgicos, radioterapia e quimioterapia, enquanto entre as pacientes mais velhas, nesses mesmos estágios, houve uma maior utilização de radioterapia isolada. No entanto, como já mencionado, evidências mostradas pela literatura apontam que pacientes idosas toleram bem terapias mais agressivas para o câncer do colo do útero, não devendo a idade apenas ser o motivo de indicação para tratamentos menos agressivos $^{11,15,17-19}$.

Em geral, as pacientes idosas apresentam maiores taxas de comorbidades moderadas a graves e pior estado nutricional, o que muitas vezes contraindica tratamentos mais agressivos ${ }^{11,17}$. Neste estudo, as idosas não foram mais acometidas por comorbidades quando comparadas com as pacientes mais jovens, porém se encontravam mais comumente com valores de IMC abaixo do considerado normal, o que pode contribuir para maior debilidade do grupo idoso, influenciando na alocação dessas pacientes em terapias menos agressivas ${ }^{11,17}$.

Estudos americanos mostram que a porcentagem de pacientes adultas com câncer do colo do útero situa-se entre $69,37 \%$ a $87,55 \%$ e para as idosas entre $12,45 \%$ a $30,63 \%^{11,15,18-19}$. No corrente estudo, $40,12 \%$ eram idosas e 59,88\% eram adultas, mostrando que, em relação à população americana, as pacientes deste estudo foram diagnosticadas mais tardiamente.

Neste estudo não foi observada uma maior incidência, com significado estatístico, de tumores não escamosos em um dos grupos estudados, porém estes foram mais frequentes em idosas de coortes americanas ${ }^{11,15}$. Algumas revisóes, no entanto, mostram que houve um aumento na incidência de adenocarcinomas, desde a década de 1970 , principalmente em mulheres com idade inferior a 35 anos de idade ${ }^{20}$.

As mulheres mais velhas, não casadas e não brancas são mais acometidas por estágios avançados da doença. Nos Estados Unidos, a incidência do câncer do colo do útero é $50 \%$ mais alta em afro-americanas quando comparadas com as brancas e 66\% mais alta em hispânicas em comparação com as não hispânicas ${ }^{11,20-21}$. No presente estudo também foi observada uma maior incidência de tumores avançados em mulheres mais velhas, quando comparadas com as mais jovens. Além disso, aquelas com 60 anos ou mais são mais comumente viúvas e as adultas mais frequentemente casadas. Neste estudo, a correlação entre raça e tumores avançados náo foi observada, possivelmente pelas características étnicas da população brasileira. Esses grupos nos quais em geral se encontram estágios mais avançados da doença são menos seguidos por testes de rastreamento. Até o momento não existem diretrizes bem estabelecidas sobre o rastreio ao câncer do colo do útero em idosas, principalmente porque existem poucos ensaios clínicos incluindo pacientes mais velhas em número suficiente. Atualmente recomenda-se uma adequada avaliação de cada paciente geriátrica para se indicar o rastreamento ${ }^{21-22}$.

A principal limitação deste estudo foi a falta de dados suficientes nos prontuários em muitas variáveis analisadas, principalmente raça, estado civil, profissão e comorbidades o que ajudou a reduzir substancialmente o tamanho da amostra, o que pode ter determinado uma diferença entre o que foi constatado no estudo e o que é observado na clínica. Náo foi objetivo deste estudo a análise de sobrevida das pacientes. O período de avaliaçáo dos prontuários foi relativamente extenso (1999 a 2010) e, durante esse tempo, ocorreram mudanças e evolução no tratamento contra o câncer do colo do útero, o que pode ter interferido nos resultados obtidos. Além disso, em muitos estadiamentos, não havia um número suficiente de pacientes para se fazer uma adequada análise estatística, o que pode ter comprometido, nesses casos, a validade dos resultados, sugerindo-se, entâo, novos estudos neste assunto, com uma maior amostra populacional.

\section{CONCLUSÃO}

Este estudo revelou que a idade é um fator que influencia na escolha do tratamento de pacientes com 
câncer do colo do útero. Quando comparadas com as pacientes adultas, pacientes com idade igual ou maior que 60 anos são menos submetidas a procedimentos cirúrgicos, radioterapia e/ou quimioterapia na doença em nível inicial, entre os estágios IB e IIA, e também em tumores localmente avançados, no estágio III. Também foi observado que pacientes idosas são diagnosticadas em estadiamentos mais avançados da doença.

A presente análise mostra a importância do câncer do colo do útero em mulheres idosas, no que concerne a sua alta frequência, ao diagnóstico em estádios mais avançados e estratégias menos agressivas de tratamento. Os dados alertam para a necessidade de programas de rastreamento e estudos clínicos direcionados a esse grupo populacional.

\section{CONTRIBUIÇÕES}

Mariana do Nascimento Vilaça contribuiu na concepção do projeto, coleta de dados, análise e revisão dos dados e de literatura e redação final do artigo. Márcio Luiz Martins Junior contribuiu na coleta de dados, análise estatística e revisão de literatura e dos dados. Luciano Rios Scherrer contribuiu na análise estatística e revisão dos dados. Angélica Nogueira-Rodrigues contribuiu na concepção do projeto, análise e revisão dos dados e redaçáo final do artigo.

\section{Declaraçáo de Conflito de Interesses: Nada a Declarar.}

\section{REFERÊNCIAS}

1. Instituto Nacional de Câncer José Alencar Gomes da Silva. Estimativa 2012: incidência de câncer no Brasil [Internet]. Rio de Janeiro: INCA; 2011 [citado 2012 jan]. p. 35-7. Disponível em: http://www.inca.gov.br/ estimativa/2012/estimativa20122111.pdf

2. Jemal A, Bray F, Center MM, Ferlay J, Ward E, Forman D. Global cancer statistics. CA Cancer J Clin. 2011;61(2):69-90.

3. Brasil. Ministério da Saúde. Departamento de Informática do SUS (DATASUS). Sistema de informaçōes sobre mortalidade 2007: taxa de mortalidade específica por neoplasias malignas - óbitos por neoplasia do colo do útero segundo unidade da federação [Internet]. Brasília: Ministério da Saúde; 2011 [citado 2012 jan]. Disponível em: http://www.datasus.gov.br

4. Silva GA, Gamarra CJ, Girianelli VR, Valente JG. Tendência da mortalidade por câncer nas capitais e interior do Brasil entre 1980 e 2006. Rev Saúde Públ. 2011;45(6):1009-18.

5. Meanwell CA. The epidemiology and etiology of cervical cancer. In: Blackledge GRP, Jordan JA, Shingleton HM, editors. Textbook of gynecologic oncology. Philadelphia: W.B. Saunders; c1991. p. 250.
6. Bosch FX, de SanjoséS. Chapter 1: Human papillomavirus and cervical cancer: burden and assessment of causality. J Natl Cancer Inst Monogr. 2003;(31):3-13.

7. Wolf JK, Ramirez PT. The molecular biology of cervical cancer. Cancer Invest. 2001;19(6):621-9.

8. de Sanjosé S, Santamaria M, Alonso de Ruiz P, Aristizabal $\mathrm{N}$, Guerrero E, CastellsaguéX, et al. HPV types in women with normal cervical cytology. In: Muñoz N, Bosch FX, Shah KV, Meheus AZ, editors. The epidemiology of cervical cancer and human papillomavirus. Lyon: International Agency for Research on Cancer; 1992. p. 75-84. (IARC scientific publications; n. 119).

9. Instituto Nacional de Câncer (Brasil). Diretrizes brasileiras para o rastreamento do câncer do colo do útero. Rio de Janeiro: INCA; 2011. 104 p.

10. Instituto Nacional de Câncer (Brasil). Programa Nacional de Controle do Câncer do Colo do Útero. Painel: Razão entre exames citopatológicos e mulheres da população - visão Estado de Minas Gerais e do município de Divinópolis [Internet]. [citado 2012 jan]. Disponível em: http://www2.inca.gov.br

11. Wright JD, Gibb RK, Geevarghese S, Powell MA, Herzog TJ, Mutch DG, et al. Cervical carcinoma in the elderly: an analysis of patterns of care and outcome. Cancer. 2005;103(1):85-91.

12. Instituto Nacional de Câncer (Brasil). Câncer do colo do útero. Rev bras cancerol. 2000;46(4):351-4. Condutas do INCA/MS.

13. Scottish Intercollegiate Guidelines Network. Management of cervical cancer: a national clinical guideline [Internet]. Edinburgh: SIGN; 2008 [cited 2012 May]. Available from: http://www.sign.ac.uk

14. Rose PG, Bundy BN, Watkins EB, Thigpen JT, Deppe G, Maiman MA, et al. Concurrent cisplatin-based radiotherapy and chemotherapy for locally advanced cervical cancer. N Engl J Med. 1999;340(15):1144-53.

15. Goodheart M, Jacobson G, Smith BJ, Zhou L. Chemoradiation for invasive cervical cancer in elderly patients: outcomes and morbidity. Int J Gynecol Cancer. 2008;18(1):95-103.

16. Morris M, Eifel PJ, Lu J, Grigsby PW, Levenback C, Stevens RE, et al. Pelvic radiation with concurrent chemotherapy compared with pelvic and para-aortic radiation for high-risk cervical cancer. N Engl J Med. 1999;340(15):1137-43.

17. Mitchell PA, Waggoner S, Rotmensch J, Mundt AJ. Cervical cancer in the elderly treated with radiation therapy. Gynecol Oncol. 1998;71(2):291-8.

18. Chen SW, Liang JA, Yang SN, Lin FJ. High dose-rate brachytherapy for elderly patients with uterine cervical cancer. Jpn J Clin Oncol. 2003;33(5):221-8.

19. Mousavi A, Karimi Zarchi M, Gilani MM, Behtash N, Ghaemmaghami F, Shams M, et al. Radical hysterectomy in the elderly. World J Surg Oncol. 2008;6:38. 
20. Holschneider $\mathrm{CH}$. Invasive cervical cancer: epidemiology, clinical features, and diagnosis [Internet]. [cited 2011 Sep]. Available from: http://www.uptodate.com/

21. Ferrante JM, Gonzalez EC, Roetzheim RG, Pal N, Woodard L. Clinical and demographic predictors of late-stage cervical cancer. Arch Fam Med. 2000;9(5):439-45.

22. Terret C, Castel-Kremer E, Albrand G, Droz JP. Effects of comorbidity on screening and early diagnosis of cancer in elderly people. Lancet Oncol. 2009;10(1):80-7. 


\begin{abstract}
Introduction: Advanced age is associated with lower rates of survival and is a major factor for selection of cancer treatment. Objectives: To evaluate the epidemiological characteristics and differences between treatments performed in elderly women (aged 60 years or older) with cervical cancer compared with those relating to adult patients, aged under 60 years. Method: Retrospective and descriptive study in which medical records of patients diagnosed with cervical cancer between 1999 and 2010 were analyzed. The records were separated into two groups: elderly, aged 60 years or older, and adults under 60 years. The data collected were saved in Microsoft Office Excel 2003 spreadsheets. The clinical and epidemiological characteristics collected were compared using chi-squared test. The p value considered as significant was less than 0.05 . Results: Among 67 elderly patients (40.12\%) and 100 adults (59.88\%), there was a higher incidence of advanced tumors IIB and III in the elderly, while the initial stages in situ, IB and IIA, were more common between adults $(\mathrm{p}<0.05)$. The adults staged in IB, IIA and III were more subjected to surgical procedures, radiotherapy and chemotherapy, while in the elderly the most common treatment was radiotherapy alone $(\mathrm{p}<0.05)$. Conclusion: This analysis shows the relevance of cervical cancer in older women, with regard to high frequency, more advanced stages at diagnosis and less aggressive treatment strategies. The data alerts to the need of screening programs and clinical studies directed to this population group.

Key words: Uterine Cervical Neoplasms/therapy; Health Profile; Aged; Retrospective Studies; Epidemiology, Descriptive
\end{abstract}

\title{
Resumen
}

Introducción: La edad avanzada está relacionada con tasas inferiores de supervivencia y es un factor importante para la selección del tratamiento del cáncer. Objetivos: Evaluar las características epidemiológicas y las diferencias entre los tratamientos en mujeres mayores, con más de 60 años o mayores, con cáncer del cuello del útero en comparación con los que se refieren a pacientes adultas, menores de 60 años. Método: Estudio retrospectivo y descriptivo donde se analizaron las historias clínicas de las pacientes diagnosticadas con cáncer del cuello del útero entre 1999 y 2010. Las historias fueron divididas en dos grupos: mujeres mayores, con más de 60 ańos o mayores, y las adultas con menos de 60 años. Los datos obtenidos fueron guardados en plantillas del programa Microsoft Office Excel 2003. Las características clínicas y epidemiológicas recogidas fueron comparadas utilizándose la prueba chi-cuadrado. El valor de p considerado significativo fue menor de 0,05. Resultados: Entre las 67 pacientes de edad avanzada $(40,12 \%)$ y 100 adultas (59,88\%), hubo una mayor incidencia de tumores avanzados IIB y III en las ancianas, mientras las etapas iniciales in situ, IB y IIA se verificaron más entre las adultas ( $<<0,05)$. Las adultas estadiadas en IB, IIA y III fueron más sometidas a procedimientos quirúrgicos, radioterapia y quimioterapia, mientras que en las ancianas el tratamiento más común fue la radioterapia aislada $(\mathrm{p}<0,05)$. Conclusión: Este análisis muestra la relevancia del cáncer del cuello del útero en las mujeres mayores, con respecto a la alta frecuencia, las fases más avanzadas al diagnóstico y estrategias de tratamiento menos agresivas. Los datos alertan para la necesidad de programas de rastreo y estudios clínicos dirigidos a este grupo de la población.

Palabras clave: Neoplasias del Cuello Uterino/terapia; Perfil de Salud; Anciano; Estudios Retrospectivos; Epidemiología Descriptiva 